\title{
Uso de la Transformada de Ondeletas (Wavelet Transform) en la Reducción de Ruidos en las Señales de Campo Eléctrico producidas por Rayos
}

\author{
Francisco Santamaría $^{(1)}$, Camilo A. Corté $\mathbf{s}^{(2)}$ y Francisco J. Román ${ }^{(2)}$ \\ (1) Universidad Distrital Francisco José de Caldas, Ingeniería Eléctrica, Grupo de Compatibilidad e \\ Interferencia Electromagnética - GCEM, Crr 7 No $40-53$, piso 5, Bogotá D.C. - Colombia \\ (2) Universidad Nacional de Colombia, Grupo de Investigación en Compatibilidad \\ Electromagnética - EMC-UN, Laboratorio de Compatibilidad Electromagnética, Edif. 411, \\ Ciudad Universitaria, Bogotá D.C. - Colombia (e-mail: fsantamariap@udistrital.edu.co)
}

Recibido Abr. 25, 2011; Aceptado Jun. 24, 2011; Versión Final recibida Ago. 24, 2011

\begin{abstract}
Resumen
Se analiza el efecto del ruido en el cálculo de parámetros temporales de las señales de campo eléctrico producidas por los rayos empleando un procedimiento basado en la transformada de ondeletas (wavelet trasform) para eliminación de ruido. Se emplearon señales registradas en Bogotá-Colombia las cuales fueron afectadas por el ruido producido por el conversor análogo digital, por el circuito electrónico y por el ruido ambiental. Se procesaron las señales de campo eléctrico para reducir el nivel de ruido presente en dichas señales usando transformada de ondeletas. La efectividad de la técnica propuesta se demostró al analizar los principales parámetros de las señales de campo eléctrico, encontrando diferencias de hasta el $84 \%$ al comparar los parámetros de las señales originales respecto de las señales procesadas. En conclusión, la transformada de ondeletas es una técnica adecuada para la reducción del ruido de las señales de campo eléctrico de los rayos.
\end{abstract}

Palabras clave: campo eléctrico de los rayos, técnicas de eliminación de ruido, transformada de ondeletas

\section{Use of the Wavelet Transform in Noise Reduction of the Electric Field Signals Produced by Lightning}

\begin{abstract}
The noise effect in determining the temporal parameters in lightning electrical field signals are analyzed using wavelet transforms for noise reduction. Signals recorded in Bogotá-Colombia which were affected by noise from the analog-to-digital converter, from the electronic circuits and from environmental noise were used. By using a wavelet based noise reduction procedure, electric field signals were processed and the level of noise in these signals was reduced. The effectiveness of the proposed technique was demonstrated by analyzing the main parameters of electric field signals, obtaining deviations up to $84 \%$ when parameters of the original signals were compared with the ones of the processed signals. In conclusion, the wavelet transform represents an appropriate technique for reducing the noise of lightning electric field signals.
\end{abstract}

Keywords: lightning electric field, noise reduction techniques, wavelet transform 


\section{INTRODUCCIÓN}

Los rayos son descargas eléctricas transitorias de alta corriente que ocurren en la atmósfera de la tierra y otros planetas. Debido al camino de la descarga, los rayos se clasifican en dos tipos: rayos nube - tierra (Cloud to Ground Flashes) y entre nubes (Intracloud Flashes). Aunque aproximadamente el $75 \%$ de los rayos se presentan dentro de la nube, son los rayos nube - tierra los que mayor peligro presentan para las personas, animales y estructuras (Rakov y Uman, 2003). De acuerdo con la polaridad de la carga que se desplaza de la nube a la tierra, los rayos nube tierra se pueden dividir en dos grupos: rayos negativos (Negative Ground Flash) y rayos positivos (Positive Ground Flash). Aproximadamente el $90 \%$ de los rayos nube - tierra son negativos mientras que el $10 \%$ restante son positivos (Rakov y Uman, 2003). La descarga negativa nube tierra inicia en la nube y transporta decenas de Culombios de carga hacia tierra. La descarga completa se conoce como "Flash", y se compone normalmente de varios pulsos de alta corriente o descargas de retorno (return strokes). En una sola descarga de rayo se presentan en promedio 5 descargas de retorno sucesivas (subsequent Strokes), cuyas corrientes pueden fluir a lo largo del mismo canal (Rakov y Uman, 2003). Cuando estos transitorios alcanzan la tierra pueden producir daños tales como interrupción del suministro de energía eléctrica, interferencia en sistemas de comunicación y deterioro de dispositivos eléctricos y electrónicos. Estos factores, sumados a la necesidad de proteger la vida humana, han llevado a que el tema de descargas atmosféricas, incluyendo la física del fenómeno y los mecanismos de protección contra rayos, se haya convertido en un área de investigación importante durante las últimas décadas (Cooray, 2003).

A partir de las mediciones de campos eléctricos se puede extraer información importante para aplicaciones en ingeniería. El campo eléctrico generado por las nubes de tormenta es importante en el estudio de protección contra rayos por tres razones: primero, estos campos eléctricos a nivel del suelo son responsables de la iniciación de líderes ascendentes (upward leader) desde las estructuras de mayor altura. Segundo, estos campos producen descargas corona en estructuras puntiagudas o afiladas, lo cual genera carga espacial que puede influir en el proceso de unión del líder descendente y el líder ascendente (lightning attachment). Tercero, estos campos pueden usarse en detectores y alarmas contra rayos (Cooray, 2010). El campo eléctrico producido por los líderes escalonados (stepped leader) a medida que viajan hacia tierra, es el responsable del inicio y propagación de los líderes ascendentes. Por lo tanto, este campo eléctrico es fundamental para determinar los posibles puntos de impacto de los rayos en las estructuras (Becerra et al., 2006).

El interés al analizar el campo eléctrico debido a la descarga de retorno es determinar de manera confiable los principales parámetros de las formas de onda, como son: el pico, el tiempo de ascenso y el tiempo de cruce por cero. Estos parámetros son necesarios para la evaluación y desarrollo de modelos de la descarga de retorno del rayo (Cooray, 2003, Hussein et al., 2008), mientras que desde el punto de vista de ingeniería, el parámetro más importante es el valor pico, ya que a partir de éste se puede estimar el valor pico de la corriente de la descarga de retorno, el cual se emplea en el diseño de los sistemas de protección contra rayos (IEC 62305-1, 2010).

Los principales parámetros de la descarga varían dependiendo de las condiciones geográficas y ambientales de cada país. La correcta estimación de estos parámetros a partir de las señales de campo eléctrico es esencial en el diseño y construcción de protecciones para líneas de potencia, telecomunicaciones y dispositivos electrónicos sensibles. En diferentes lugares del mundo se han llevado a cabo en las últimas décadas múltiples mediciones de las señales de los campos eléctricos de los rayos, las cuales han permitido avanzar en el conocimiento de sus principales características (Fernando y Cooray, 2007; Nag, 2009; Ahmad et al., 2010). Sin embargo, la obtención de estos parámetros a partir de las formas de onda del campo eléctrico o la corriente de la descarga de retorno no es una tarea sencilla debido a la existencia de diferentes tipos de ruido en las señales registradas (Baba et al., 2004; Gou et al., 2009; Nedjah et al., 2010). En algunos casos si se conoce la frecuencia del ruido éste puede reducirse empleando filtros análogos 0 mediante el procesamiento de las señales medidas. En el caso de filtros su uso ha sido ampliamente probado en diferentes aplicaciones, ya que tienen la ventaja de ser estables ante cambios en las condiciones ambientales, además de que la tecnología electrónica ha evolucionado rápidamente, lo que los hace pequeños y confiables. Sin embargo, estos filtros 
basan su funcionamiento en la reducción de señales en frecuencias específicas, por lo que junto con las señales de ruido se puede perder información de interés (Sonnadara et al., 2006).

La señales de campos eléctricos debidos a los rayos son señales no estacionarias, las cuales se pueden procesar mediante herramientas matemáticas tales como la Transformada de Fourier (Nedjah et al., 2010). En este caso, la señal se divide en segmentos de tiempo en los cuales se puede considerar como cuasi-estacionaria, con lo que la Transformada de Fourier se puede aplicar en cada segmento de la señal. Aunque la Transformada de Fourier es una técnica ampliamente conocida y tiene algunas ventajas frente a otras técnicas matemáticas, también tiene algunas desventajas. Con esta transformada sólo se conoce en qué intervalo de tiempo se produce una singularidad, pero no es posible distinguir diferentes eventos dentro de una misma ventana, por lo que éstos no pueden discriminarse si aparecen muy cerca unos de otros. Cuando una señal temporal se transforma al dominio de la frecuencia mediante la Transformada de Fourier parte de la información se pierde, lo que no es muy importante cuando la señal es continua o repetitiva, pero cuando la señal es transitoria como las señales de campo eléctrico de los rayos, la pérdida de información puede ser significativa.

En las últimas décadas la Transformada Wavelet se ha venido utilizando cada vez más en problemas de reconocimiento de patrones, procesamiento y síntesis de señales de diferentes tipos, análisis de imágenes, compresión de grandes volúmenes de información, eliminación de ruido de diferentes tipos de señales, y otros casos (Addison, 2002; Martínez et al, 2010). La Transformada Wavelet, a diferencia de la transformada de Fourier, proporciona simultáneamente una representación en el tiempo y la frecuencia de una señal dada, por lo que es apropiada para el análisis de señales donde se necesita tener resolución de tiempo-frecuencia, tales como las señales de campo eléctrico de los rayos (Astafeva, 1996; Canal, 2008). En los últimos años la Transformada Wavelet ha sido empleada para analizar las señales de rayos. Sheshyekani et al (2006) utilizaron un caso particular de la Transformada Wavelet discreta, la Wavelet Haar, para identificar la ocurrencia de una descarga de retorno del rayo. Miranda (2008) y Sharma et al (2010) utilizaron la Transformada Wavelet para evaluar el espectro de potencia de los diferentes eventos de los rayos a partir de mediciones del campo eléctrico. Gou et al (2009) estudiaron el comportamiento fractal de las formas de onda del campo eléctrico producidas por la primera descarga de retorno empleando una técnica Wavelet multiresolución.

Islam et al (2009) aplicaron un método de sustracción espectral STFT en la eliminación del ruido de las señales de la derivada de la corriente del rayo registradas en la Torre Nacional de Canadá (CN Tower). En la publicación se evaluó la relación señal a ruido antes y después de aplicar el procedimiento de filtrado de las señales y se concluyó que los resultados obtenidos eran satisfactorios. Nedjah et al (2010) investigaron varias técnicas adaptativas (incluida la transformada Wavelet) para la eliminación del ruido de las mismas señales de la derivada de la corriente del rayo empleadas por Islam (2009), además de las señales de corriente, encontrando que ninguna de las técnicas estudiadas se adaptó perfectamente a la naturaleza transitoria de las señales registradas, por lo que propusieron una nueva técnica que sí se ajustó a sus requerimientos. Sin embargo, en dicho trabajo se contaba con una caracterización del ruido existente en las señales medidas.

Este artículo presenta la aplicación de la Transformada Wavelet en la eliminación del ruido de las señales de campo eléctrico de los rayos y su efecto en la evaluación de los principales parámetros de las señales registradas. En este sentido, inicialmente se describe brevemente el sistema de medida. En esa misma sección se presentan los parámetros de la forma de onda del campo eléctrico que se requiere evaluar y el efecto del ruido en la estimación de dichos parámetros. Luego se describe la Transformada Wavelet que se emplea en la eliminación del ruido y se presentan los resultados de la aplicación de la Transformada Wavelet en la reducción del ruido; en esta parte se observa que al emplear esta técnica se pueden determinar parámetros que antes eran difíciles de evaluar. Posteriormente se presenta una sección de discusión en la que se comparan los resultados obtenidos antes y después de emplear la técnica de eliminación de ruido. Finalmente, se concluye resaltando las ventajas de la Transformada Wavelet en el procesamiento de las señales de campo eléctrico de los rayos. 


\section{MEDICIONES DEL CAMPO ELÉCTRICO DE LOS RAYOS}

Este artículo se basa en mediciones del campo eléctrico de los rayos realizadas en Bogotá D.C. $\left(4^{\circ} 36^{\prime} \mathrm{N}, 74^{\circ} 5^{\prime} \mathrm{W}, 2600 \mathrm{~m}\right.$ sobre el nivel del mar). Durante la campaña de medición se registraron sesenta y nueve señales de descargas de rayos nube-tierra. De éstas, 68 fueron negativas y solamente una positiva. De las descargas negativas, 41 tenían una descarga de retorno, mientras que 27 presentaron descargas de retorno sucesivas. La media aritmética del número de descargas de retorno por rayo fue de 1.8 (Santamaría et al., 2006).

\section{Sistema de medida}

El sistema de medida empleado en la medición del campo eléctrico de los rayos se compone de una antena de placas paralelas con un diámetro de $0.45 \mathrm{~m}$ y una separación entre placas de 0.03 m.; un cable coaxial de $50 \Omega$ y $0.6 \mathrm{~m}$ por el que se transmite la señal desde la antena hasta el circuito electrónico, el cual se compone principalmente de un amplificador búfer y un atenuador de -20 dB y un cable coaxial de $50 \Omega$ y $20 \mathrm{~m}$ por el que se transmite la señal desde el circuito electrónico hasta el osciloscopio. Este último empleado para registrar las señales con un ancho de banda de $500 \mathrm{MHz}$ y velocidad de muestreo de $1 \mathrm{GS} / \mathrm{s}$. Las mediciones se realizaron con una ventana de $500 \mathrm{~ms}$ y una resolución de 1 dato cada 500 ns.

\section{Características de los campos eléctricos radiados}

La forma de onda de los campos eléctricos generados por las descargas de retorno de los rayos es importante para entender la física de la descarga y los mecanismos a partir de los cuales se produce el encuentro entre el líder descendente y un líder ascendente. Además, los campos radiados proporcionan información con respecto a la variación temporal de la corriente de la descarga de retorno y los voltajes que pueden inducirse en instalaciones eléctricas cuando los campos electromagnéticos generados por los rayos interactúan con ellas (Bermudez et at, 2007; Ren et al, 2008). Cooray (2003) resume las principales características de los campos eléctricos radiados por descargas de retorno negativas. La parte inicial de la señal presenta una transición lenta ( $\mathrm{F}$ en la Fig. 1), que para la primera descarga de retorno puede durar aproximadamente $5 \mu \mathrm{s}$ y alrededor de $0.5 \mu$ s para descargas de retorno sucesivas, seguida de una transición mucho más rápida hasta el pico $(R)$. El tiempo de esta parte de la señal, $10 \%-90 \%$, normalmente se encuentra alrededor de $0.1 \mu \mathrm{s}$. El frente lento puede ser generado por el incremento exponencial de la corriente en el líder ascendente a medida que éste se mueve a través de la región del streamer del líder descendente, es decir, después del encuentro del líder ascendente con el líder descendente. Este estado podría ser interpretado como el estado inicial de la descarga de retorno. La transición rápida en la señal de campo eléctrico corresponde a un rápido incremento en la corriente cuando el líder ascendente alcanza el núcleo caliente del canal del líder descendente.

A medida que el campo eléctrico decrece puede presentar unos picos secundarios (a y b en la Fig. 1) separados entre sí algunas decenas de microsegundos, que se deben a rápidas variaciones en la corriente y la velocidad cuando el frente de la descarga de retorno alcanza ramificaciones del canal del líder descendente. El tiempo de cruce por cero para la primera descarga de retorno normalmente está en el rango de 50 a $100 \mu \mathrm{s}$, y para descargas sucesivas este valor oscila entre 30 y $50 \mu \mathrm{s}$. Este parámetro depende de la rapidez con que la corriente generada en el proceso de neutralización decrece a lo largo del canal: si esta corriente decrece muy rápido el tiempo de cruce por cero será más corto, y si por el contrarío la caída de la corriente es lenta, el tiempo de cruce por cero será mayor. La forma en la que esta corriente decrece a lo largo del canal depende en cierta manera de la distribución de carga en el canal del líder. Adicionalmente, el tiempo de cruce por cero depende de la longitud vertical del canal, la cual es aproximadamente igual a la altura del centro de carga negativo en la nube.

La Fig. 1 presenta una de las señales de campo eléctrico registrada durante la campaña de medición. En esta figura se identifican algunas de las características descritas anteriormente. Además, se observa el ruido a lo largo de toda la forma de onda, el cual reduce la confiabilidad de los valores obtenidos para algunos de los parámetros señalados, en particular los que ocurren en 
tiempos muy cortos o que tienen una magnitud comparable con el nivel de ruido. La pequeña señal marcada con $L$ parece corresponder a un pulso típico debido a los pasos del líder descendente previo a la formación y desarrollo de la descarga de retorno. Sin embargo, el nivel de ruido en esta parte de la onda es comparable con la señal y hace difícil su análisis. Para determinar la duración del frente lento $(F)$ es necesario ubicar el cero de la señal, lo cual no se puede hacer con precisión debido al nivel de ruido. Determinar el valor pico y la magnitud de los picos secundarios ( $a$ y $b$ ) no presenta dificultad. El tiempo de cruce por cero se puede calcular con la señal tal como aparece en la Fig. 1. No obstante, a medida que ese tiempo se hace menor el error se hace mayor, ya que nuevamente se requiere conocer el cero de la señal a partir del cual se determinaría dicho tiempo.

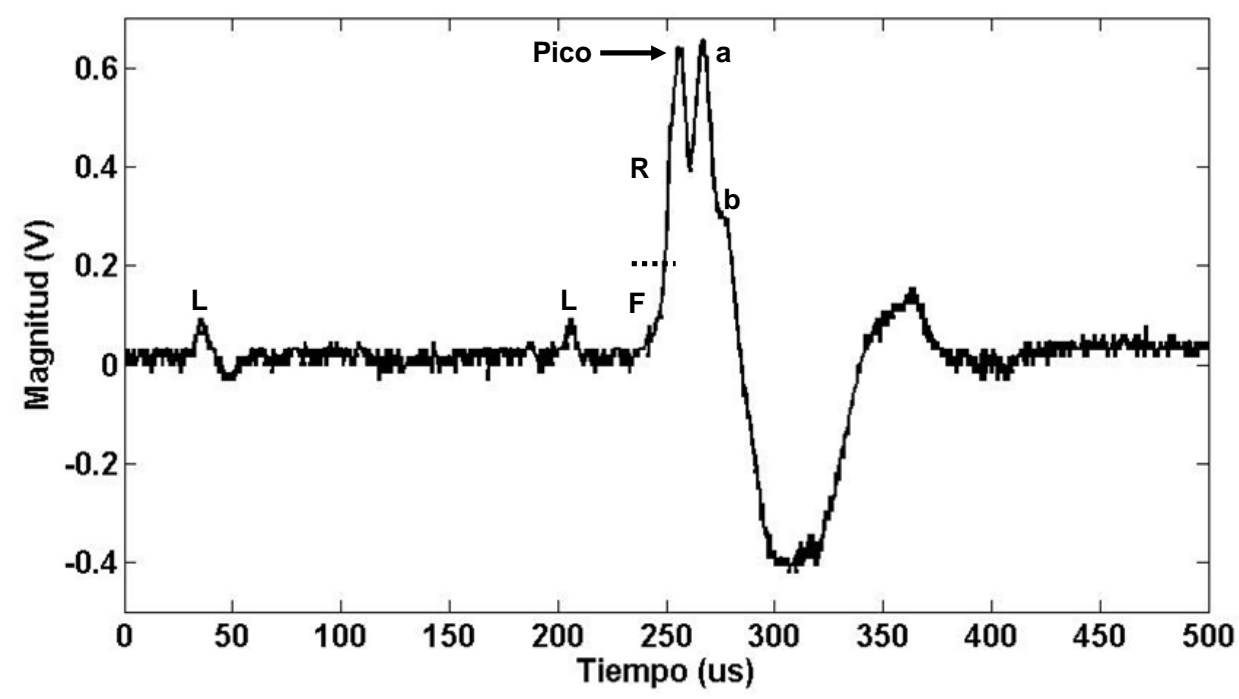

Fig. 1: Onda de campo eléctrico producido por una descarga de retorno nube - tierra negativa

\section{Fuentes de ruido}

Los sistemas de medida pueden verse afectados por fenómenos no deseados, tales como ruido electrónico y factores externos que deterioran la calidad de las señales medidas. Esto depende de las variables de interés y la sensibilidad de los sistemas de medida. En ocasiones este ruido puede reducirse controlando el ambiente en el que se encuentra el sistema de medida o, en la medida en que se conocen las características del ruido, mediante el uso de filtros análogos o el procesamiento de las señales medidas. Algunas de las posibles fuentes de ruido son:

Debido al conversor análogo - digital (ADC): Cuando se digitaliza una señal, el número de bits usados para representar la medición ( 8 bits en este caso) determina la máxima relación posible señal - ruido. Esto se debe a que el nivel mínimo de ruido es el error causado por la cuantificación de la señal (procesamiento digital), conocido como ruido de cuantificación (quantization noise). Este nivel de ruido es no lineal, depende del tipo de señal y se puede modelar como una señal de error análoga sumada con la señal real, es decir la señal antes del procesamiento digital. Debido al circuito electrónico: Todo circuito electrónico tiene una señal característica no deseada llamada ruido electrónico. Este ruido varía dependiendo del circuito y puede producirse debido a diferentes efectos, tales como ruido térmico, ruido de disparo (trigger noise) y calidad de fabricación. Debido a ruido ambiental: En los últimos años el ruido electromagnético ambiental ha venido aumentando. Algunos ejemplos de este tipo de ruido son el ruido procedente de dispositivos como televisores, transmisores de radio y telefonía móvil, de máquinas eléctricas y de fenómenos naturales como los rayos, entre otros. En las mediciones realizadas en Bogotá, los cables coaxiales empleados tenían doble pantalla electromagnética, una de las técnicas comúnmente empleadas para reducir los efectos del ruido electromagnético ambiental.

Algunos dispositivos no son afectados por señales de ruido debido a que en ocasiones estas señales se atenúan o distorsionan durante su viaje. Otros, por el contrario, pueden recibir estas 
señales no deseadas y operar de manera indeseable. En el caso de las mediciones del campo eléctrico de los rayos, emisiones no controladas pueden afectar el espectro de las señales registradas. Por lo tanto, es importante determinar los niveles de ruido y sus características para poder tomar medidas que permitan reducir su impacto en las señales de interés. Para el caso de las señales de campo eléctrico empleadas en este artículo se determinó que las principales fuentes de ruido fueron los circuitos electrónicos empleados en la adquisición y procesamiento digital de las señales.

\section{PROCESAMIENTO DE LAS SEÑALES}

La Transformada Wavelet de una función $x(t)$ corresponde a la descomposición de dicha función en un grupo de funciones $\Psi_{m, n}(t)$ llamadas "Wavelets" (Walker, 2008). La Transformada Wavelet Discreta (DWT) de una señal continua se puede definir como (Addison, 2002):

$$
\mathrm{DWT}_{\Psi} x(m, n)=\int_{-¥}^{¥} x(t) \Psi_{m, n}^{*}(t) d t
$$

Las wavelets son generadas a partir de la traslación y cambio de escala de una misma función wavelet $\Psi_{m, n}(t)$, llamada la "Wavelet madre", definida como:

$$
\Psi_{\mathrm{m}, \mathrm{n}}(\mathrm{t})=\left|\mathrm{a}_{0}\right|^{-\mathrm{m} / 2} \Psi\left(\frac{\mathrm{t}-\mathrm{nb} \mathrm{b}_{0} \mathrm{a}_{0}^{\mathrm{m}}}{\mathrm{a}_{0}^{\mathrm{m}}}\right)
$$

Donde $n$ y $m$ son los parámetros para discretizar la traslación y cambio de escala de la función, $a_{0}$ es un parámetro de paso de dilatación fijo específico, y $b_{0}$ es el parámetro de localización.

\section{Reducción de ruido usando teoría Wavelet}

El objetivo al realizar cualquier proceso de este tipo es eliminar el ruido presente en los datos provenientes de una medición de la manera más eficiente posible, preservando las características fundamentales de la señal tratada (Ma et al, 2002). La Transformada Wavelet se ha empleado en la eliminación de ruido en diferentes campos y ha demostrado ser superior a las técnicas tradicionales de eliminación de ruido que emplean filtros pasa bajos. En este caso la eliminación de ruido se lleva a cabo sin remover componentes de la señal con frecuencias dentro de la banda de corte del filtro y que pueden tener algún significado físico dentro de la señal.

Los métodos de eliminación de ruido basados en la Transformada de Fourier se emplean comúnmente en el domino de la frecuencia, pero la Transformada de Fourier no proporciona información en el dominio del tiempo, como por ejemplo el tiempo en el que ocurren los cambios en la frecuencia (Ma et al, 2002). Las señales de campo eléctrico de los rayos son señales transitorias no periódicas que no son fáciles de caracterizar con las transformadas convencionales. La técnica más conocida se llama umbral de eliminación de ruido wavelet (Wavelet thresholding denoising), la cual tiene la ventaja de que permite seleccionar automáticamente un umbral sin tener conocimiento previo de la señal (Walker, 2008). Esta técnica fue empleada para el desarrollo de este trabajo. La Fig. 2 muestra un diagrama de bloques que explica la técnica usada.

Inicialmente, se selecciona una wavelet madre adecuada para realizar la descomposición de la señal de campo eléctrico en wavelets. Esta selección se puede basar en alguna de las siguientes tres posibilidades: inspección visual de la señal bajo estudio, en una correlación cruzada $\gamma$ entre la señal de interés y la wavelet madre seleccionada, o en la energía acumulada en los intervalos en donde se presentan los picos de la señal. En este trabajo se seleccionó el procedimiento de correlación cruzada, el cual maximiza la correlación de las señales de interés y las formas de onda de las wavelets madre más conocidas (Ma et al, 2002), de acuerdo con la ecuación 3 , en donde $X$, $Y$ son los datos y $\bar{X}, \bar{Y}$ los valores medios de $X$ y $Y$. 


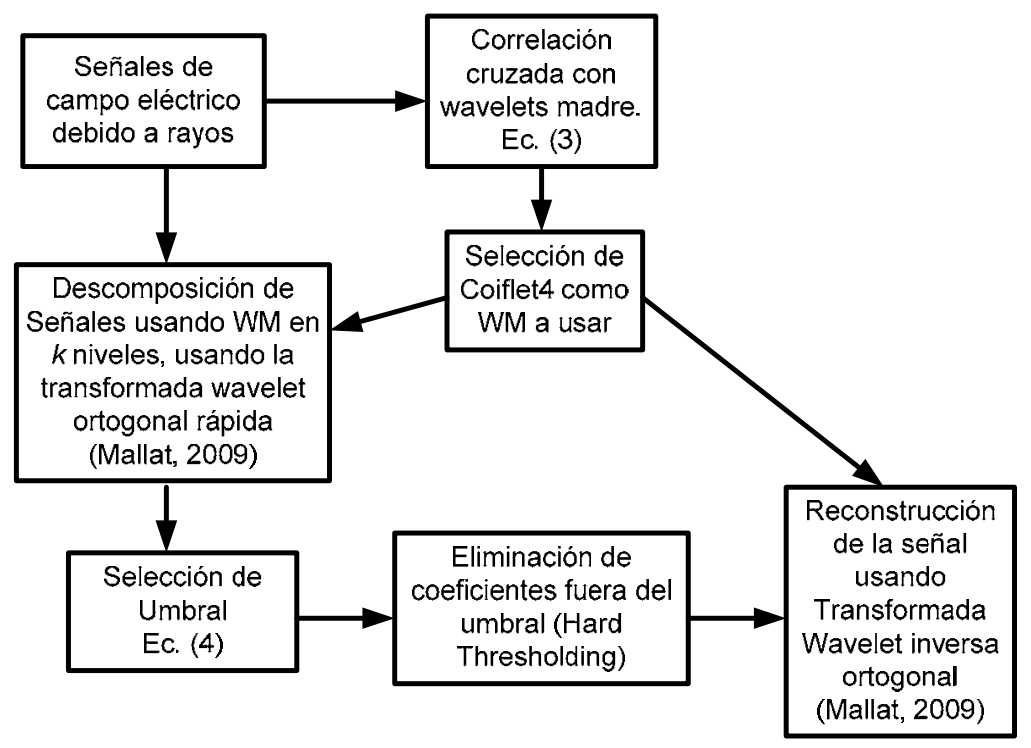

Fig. 2: Diagrama de bloques explicativo de la aplicación de la técnica de reducción de ruido usada

La wavelet madre adecuada para el tratamiento de las señales de campo eléctrico de los rayos, se seleccionó de un grupo de 24 ondas conocidas pertenecientes a las siguientes familias: Haar, Daubechies, Symlet, Coiflet y Meyer discreta.

$$
\mathrm{Y}=\frac{\sum(\mathrm{X}-\overline{\mathrm{X}})(\mathrm{Y}-\overline{\mathrm{Y}})}{\sqrt{\sum(\mathrm{X}-\overline{\mathrm{X}})^{2} \sum(\mathrm{Y}-\overline{\mathrm{Y}})^{2}}}
$$

Empleando 5 de los registros de campo eléctrico, tal como el que se muestra en la Fig. 3, y aplicándoles la ecuación 3 se encontró que los máximos factores de correlación se obtuvieron para Symlet7, Daubechies2, Coiflet3 y Coiflet4, siendo esta última la que presentó mejores resultados, por lo que fue la seleccionada para este trabajo (Fig. 4).

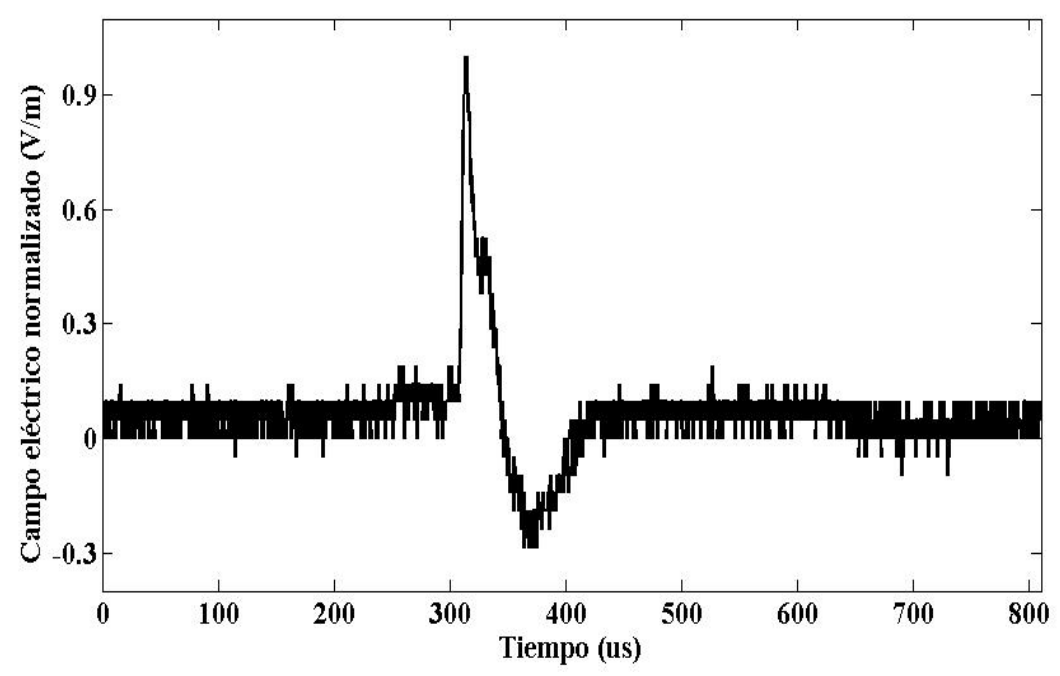

Fig. 3: Señal registrada de una descarga de retorno sucesiva (Col1) típica con alto contenido de ruido

Después se aplicó la transformada wavelet a la señal con ruido para producir los coeficientes wavelet de diferentes escalas, teniendo en cuenta que un número elevado de niveles puede mejorar el proceso de eliminación del ruido, pero a la vez si este número es excesivo puede 
reducir la señal de interés. Las pruebas realizadas mostraron que usando 5 niveles el ruido se removió correctamente de las señales de campo eléctrico (Fig. 5a).

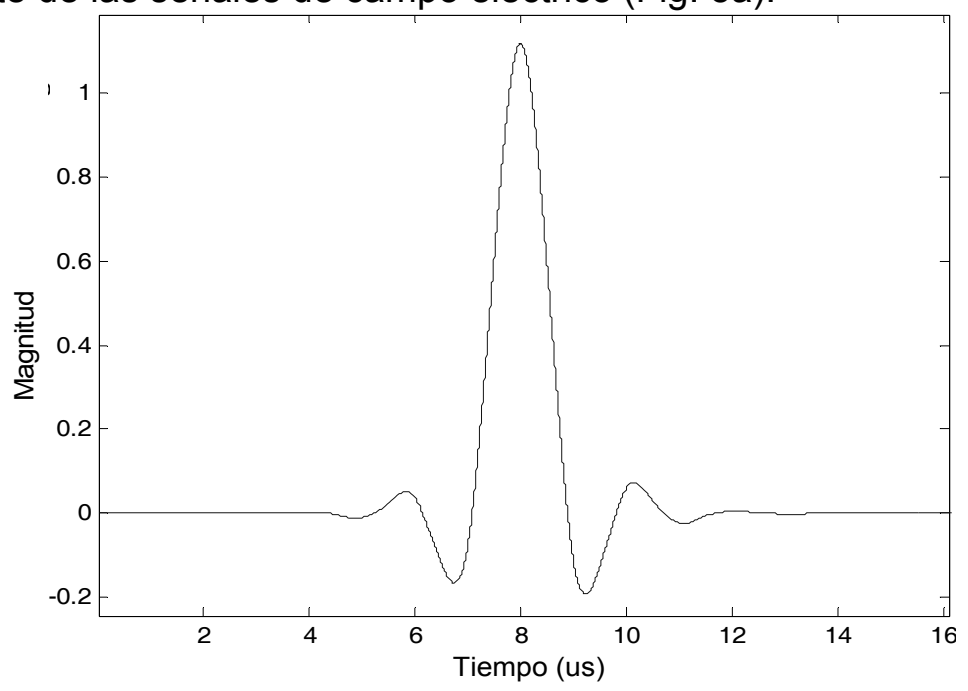

Fig 4: Wavelet madre Coiflet4 usada en el procesamiento de las señales

Para cada nivel, se seleccionó el umbral apropiado. En la literatura se encuentran varias formas de seleccionar el umbral, tales como umbrales fijos y la regla minmax, pero estos son solo adecuados para eliminar ruido blanco. Sin embargo, Donoho (1995) propone un método en el cual la estimación del umbral es basado en un procedimiento robusto independiente al nivel de ruido, el cual es el usado en este trabajo:

$\operatorname{Thr}_{\mathrm{j}}=\mathrm{m}_{\mathrm{j}} / 0.6745 \cdot \sqrt{2 \cdot \log (\mathrm{n})}$

Donde $T h r_{j}$ indica el valor del umbral al nivel $j$ de descomposición, $m_{j}$ es el valor medio de los coeficientes del nivel $j$ y $n$ es la longitud de la señal original bajo análisis.

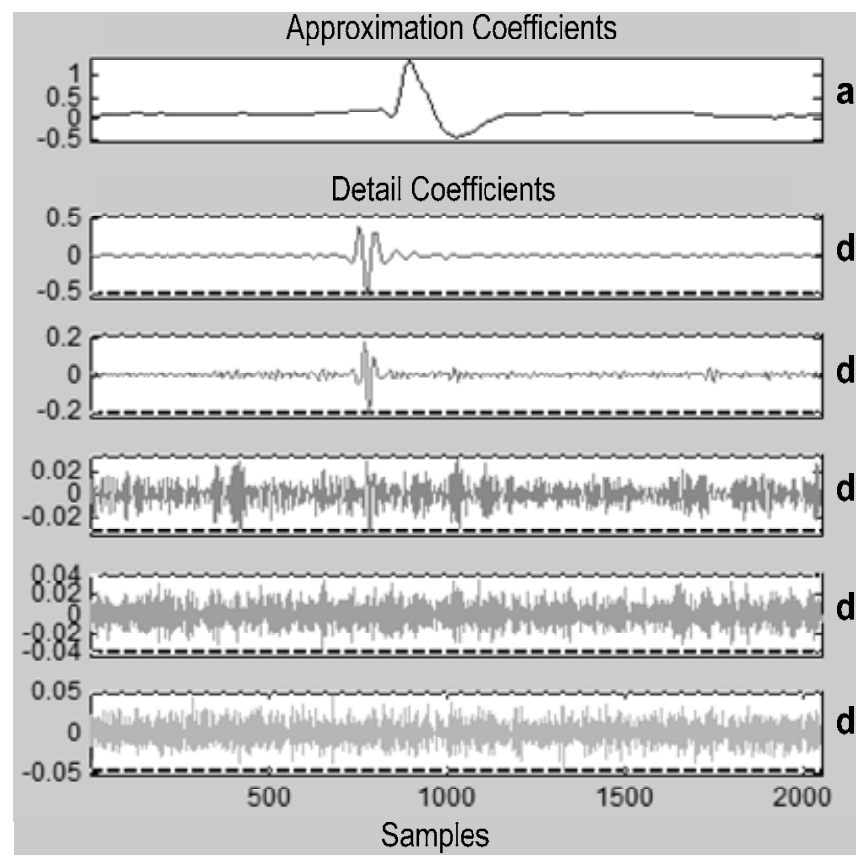

(a)

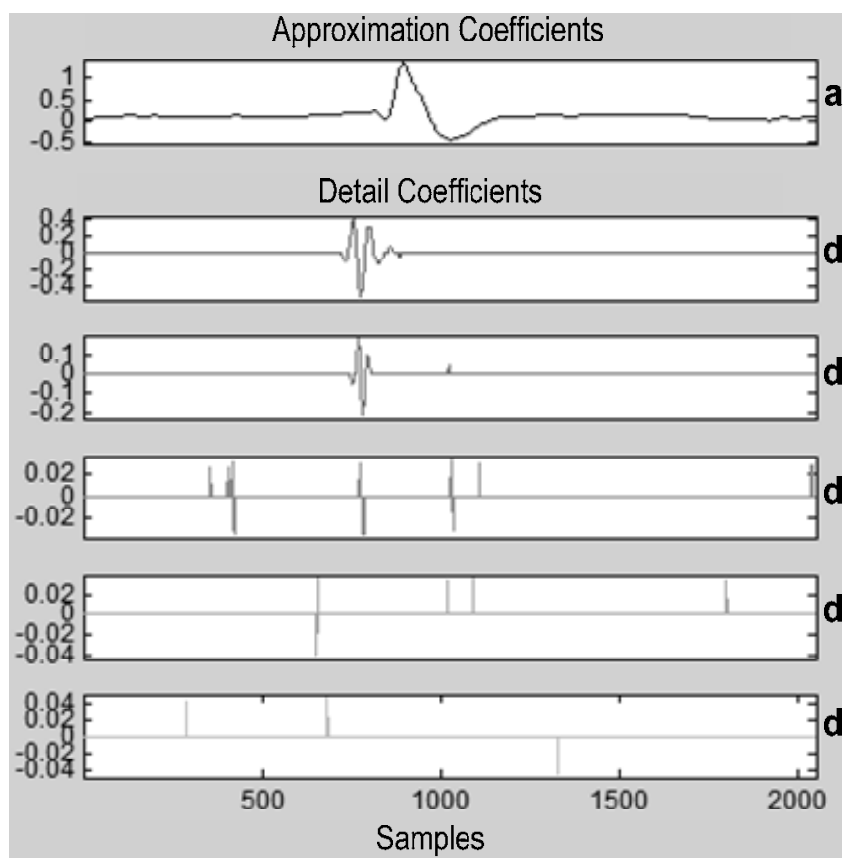

(b)

Fig 5: Aproximación y detalle de los coeficientes después de la descomposición, a. Sin umbral, b. Después de aplicar el método de umbral duro 
Además, existen dos métodos para aplicar el umbral, el duro y el suave. El duro es el más sencillo, ya que reemplaza por cero todos los coeficientes que tienen una magnitud menor que el umbral seleccionado y deja los coeficientes restantes sin tratamiento. La técnica de umbral suave reduce estos últimos coeficientes teniendo en cuenta algunos criterios adicionales (Donoho, 1995). En este trabajo se aplicó el método de umbral duro para la remoción del ruido (Fig. 5b).

Finalmente, se aplicó la Transformada Wavelet Inversa a los coeficientes wavelet para obtener las señales de campo eléctrico sin ruido.

\section{RESULTADOS}

Los resultados mostrados fueron obtenidos usando rutinas de las cajas de herramientas de Matlab Wavelet y WaveLab, así como rutinas desarrolladas por los autores. La forma de onda de la Fig. 3 representa el caso típico en el que la señal de campo eléctrico del rayo está superpuesta por un nivel de ruido. La Fig. 6 muestra el resultado obtenido al aplicar el procedimiento descrito de eliminación de ruido a partir de la transformada wavelet.

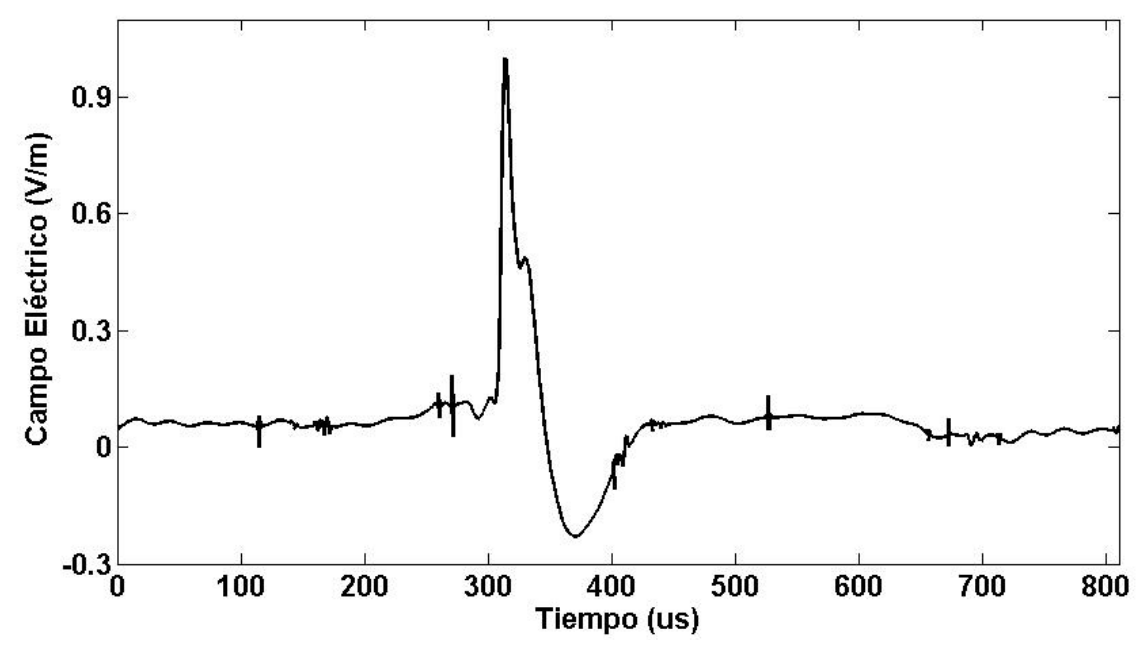

Fig 6: Señal de campo eléctrico de la Fig. 3 después de eliminado el ruido

Las señales de las figuras 7,8 y 9 son otros ejemplos de la aplicación de la metodología propuesta. En estas figuras se evidencia una mejor visualización de las características de las descargas, principalmente de los parámetros temporales del campo eléctrico. En todos los casos el eje horizontal es tiempo en microsegundos y el eje vertical es el campo eléctrico en $\mathrm{V} / \mathrm{m}$.

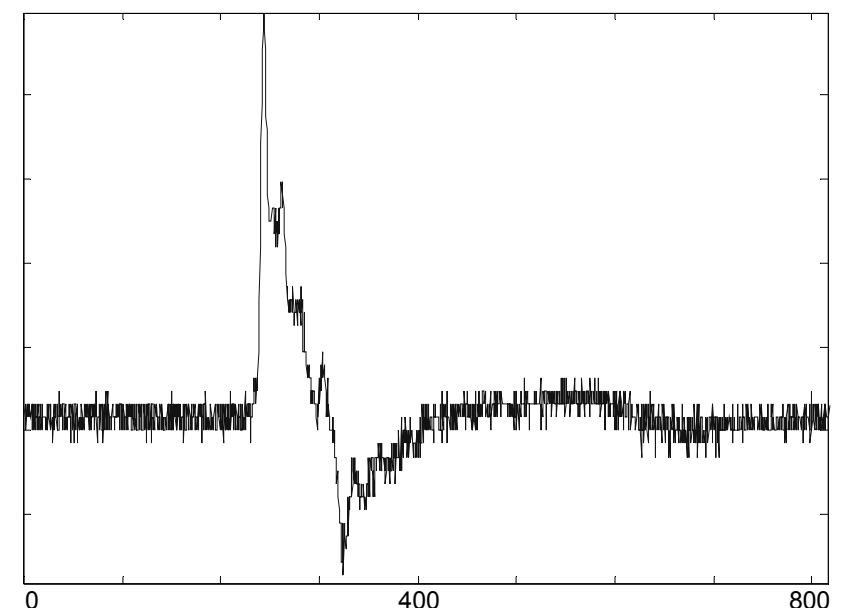

(a)

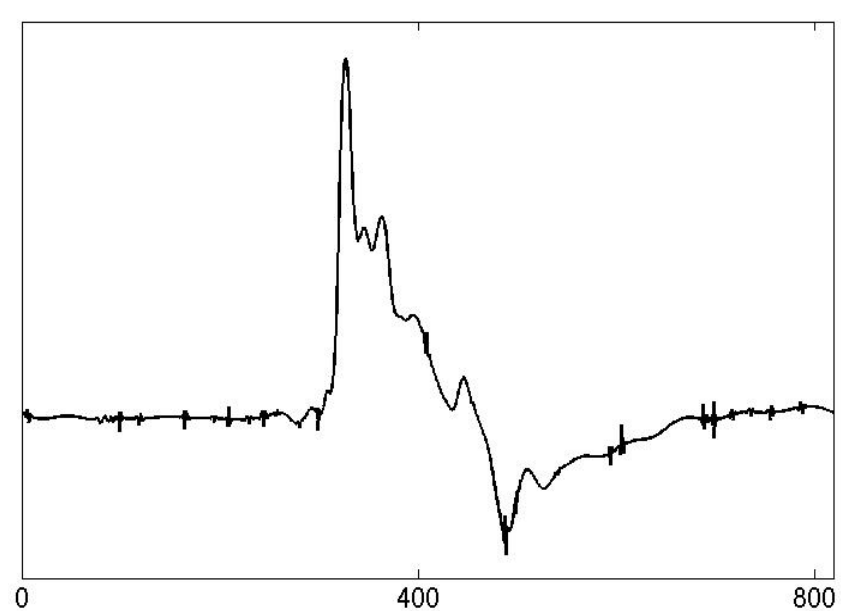

(b)

Fig 7: Campo eléctrico de una descarga de retorno (Col2), a. Señal registrada, b. Señal sin ruido 


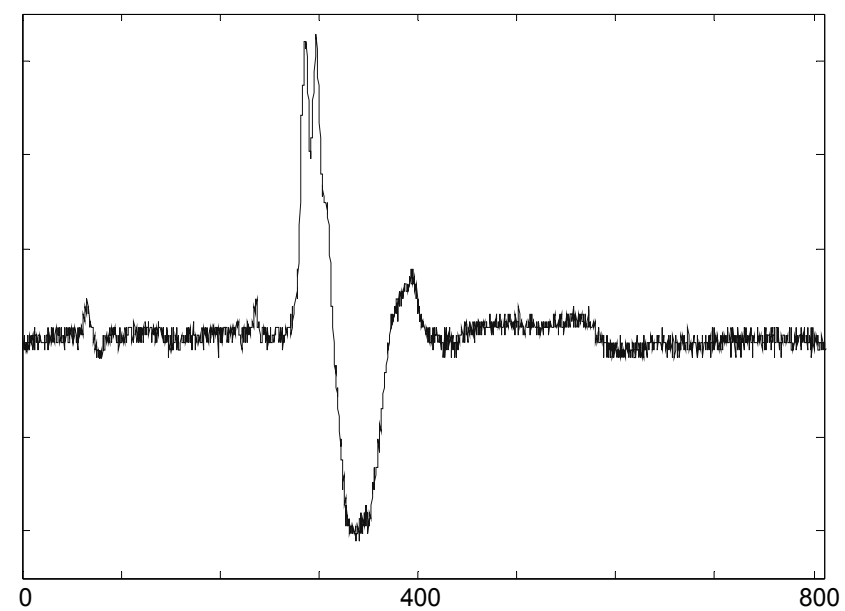

(a)

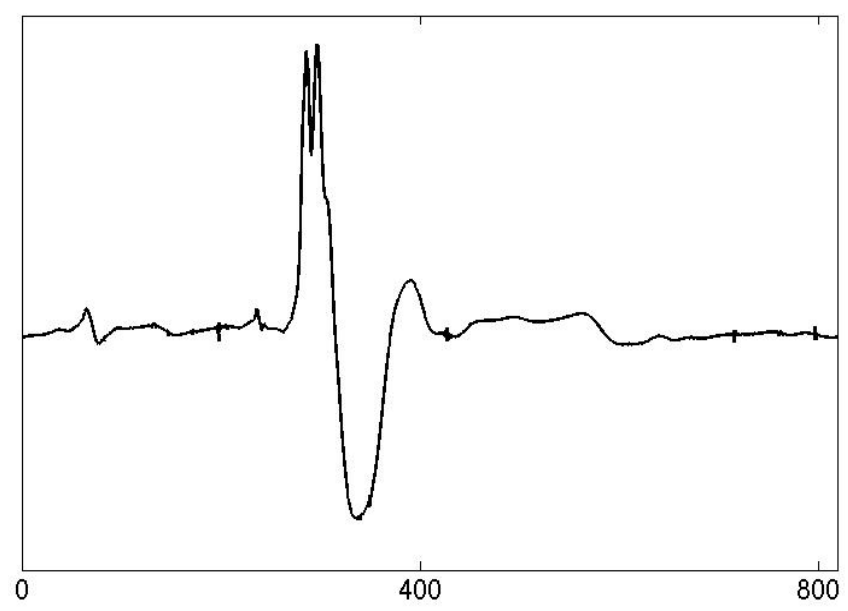

(b)

Fig 8: Campo eléctrico de una descarga de retorno (Col3), a. Señal registrada, b. Señal sin ruido

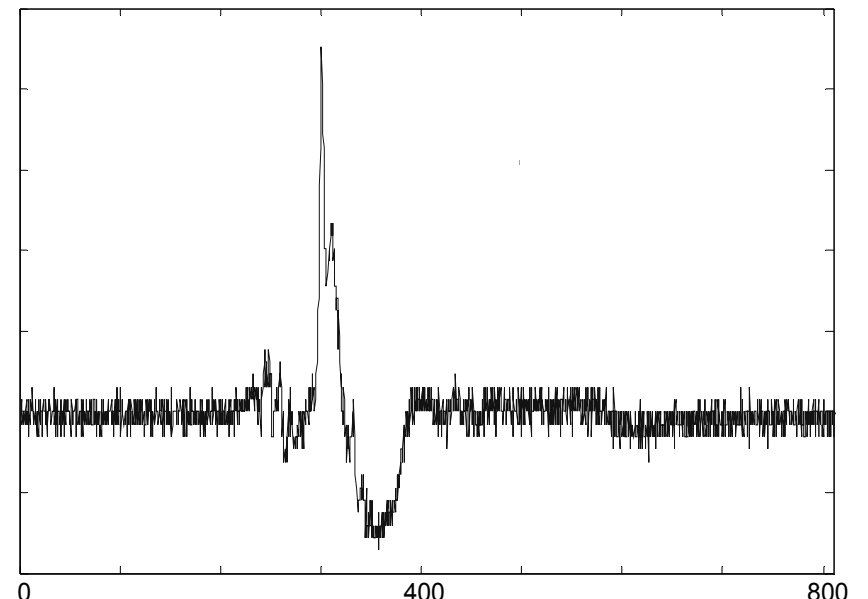

(a)

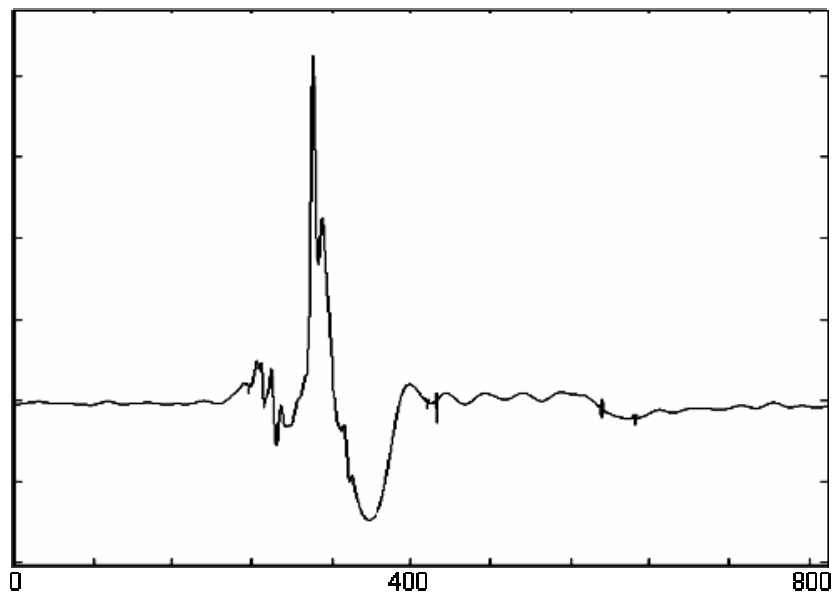

(b)

Fig 9: Campo eléctrico descarga de retorno sucesiva (Col4), a. Señal registrada, b. Señal sin ruido

\section{DISCUSIÓN}

La estimación de las principales características de la corriente de rayo a partir de las señales de campos eléctricos y magnéticos es uno de los aspectos de mayor interés en la investigación en rayos. Los principales parámetros a evaluar son los siguientes: los tiempos de ascenso de la señal (frente lento y frente rápido), el pico de la señal y el tiempo de cruce por cero. Adicionalmente, es de interés conocer la máxima derivada del campo eléctrico $(\partial E / \partial t)_{\max }$, la cual es un parámetro útil en la estimación de la variación máxima de la corriente del rayo.

La Tabla 1 presenta la comparación entre los parámetros observados antes y después del procesamiento. En esta tabla se observa que los tiempos del frente rápido y del frente lento calculados a partir de las señales procesadas presentan diferencias porcentuales entre el $3 \%$ y el $35 \%$ con respecto a los obtenidos a partir de las señales originales. Dado que dichos tiempos están asociados a la formación del rayo, estas diferencias pueden producir errores en el modelado y estimación de las características de los rayos en una región determinada. En cuanto al tiempo de cruce por cero de la señal y el valor pico del campo eléctrico, no se presentan variaciones significativas en los resultados obtenidos antes y después del procesamiento. En el caso del cruce por cero la diferencia es prácticamente cero, mientras que para el valor pico esta diferencia está entre el 1 y el $4 \%$, siendo aceptable en todos los casos. En la estimación del valor máximo de la derivada del campo eléctrico se observan las mayores diferencias entre los valores tomados de la señal procesada y los tomados de la señal original, alcanzándose diferencias de hasta el $84.09 \%$. 
Esto se debe a que las componentes de alta frecuencia de la señal de ruido se vuelven predominantes al realizar el proceso de derivación de la señal de campo eléctrico.

Tabla 1: Comparación de los parámetros de las señales de campo eléctrico de los rayos medidas en Colombia e identificadas como Col1, Col2, Col3 y Col4 antes y después de retirar el ruido *Se calculó el tiempo de ascenso (10 - $90 \%)$

\begin{tabular}{|l|c|c|c|c|c|}
\hline Señal & $\mathrm{F}(\mu \mathrm{s})$ & $\mathrm{R}(\mu \mathrm{s})$ & $\mathrm{CZ}(\mu \mathrm{s})$ & $\mathrm{Emax}(\mathrm{V} / \mathrm{m})$ & $(\partial E / \partial t)_{\max }(\mathrm{V} / \mathrm{m} / \mu \mathrm{s})$ \\
\hline Col1 & & & & & \\
Original & $4.8^{*}$ & $\mathrm{~N} / \mathrm{l}$ & 36.4 & 6.81 & 3.24 \\
Procesada & $4.3^{*}$ & $\mathrm{~N} / \mathrm{l}$ & 38.0 & 6.56 & 1.76 \\
\hline Col2 & & & & & \\
Original & 8.0 & 2.8 & 78.4 & 10.38 & 3.24 \\
Procesada & 7.6 & 2.9 & 78.4 & 10.14 & 2.45 \\
\hline Col3 & & & & & 3.94 \\
Original & 10.4 & 3.8 & 43.2 & 13.30 & 2.40 \\
Procesada & 9.6 & 2.8 & 43.2 & 13.17 & 3.24 \\
\hline Col4 & & & & & 2.12 \\
Original & 11.2 & 2.0 & 34.0 & 9.41 & 9.35 \\
Procesada & 10.8 & 1.8 & 34.0 & & \\
\hline
\end{tabular}

Para el caso de la señal Col1, no fue posible discriminar entre tiempo del frente rápido y el tiempo del frente lento, por lo que se presenta el tiempo de ascenso de la señal, calculado como el tiempo entre el 10 y el $90 \%$ del pico de la señal.

Para verificar la utilidad del método empleado para el filtrado de las señales se realizó el mismo procedimiento empleando el método clásico de Fourier para el filtrado del ruido existente en una de las señales bajo estudio. La Fig. 10 presenta una comparación entre la señal Col2 filtrada empleando la transformada wavelet descrita en este artículo y empleando la transformada de Fourier. En esta figura se observa que el método de eliminación de ruido basado en la transformada wavelet tiene un mejor desempeño que el basado en la transformada de Fourier, ya que esta última introduce oscilaciones no deseadas típicas de los métodos clásicos de filtrado de señales, causando dificultades en la interpretación de las variables de interés (Donoho, 1995).

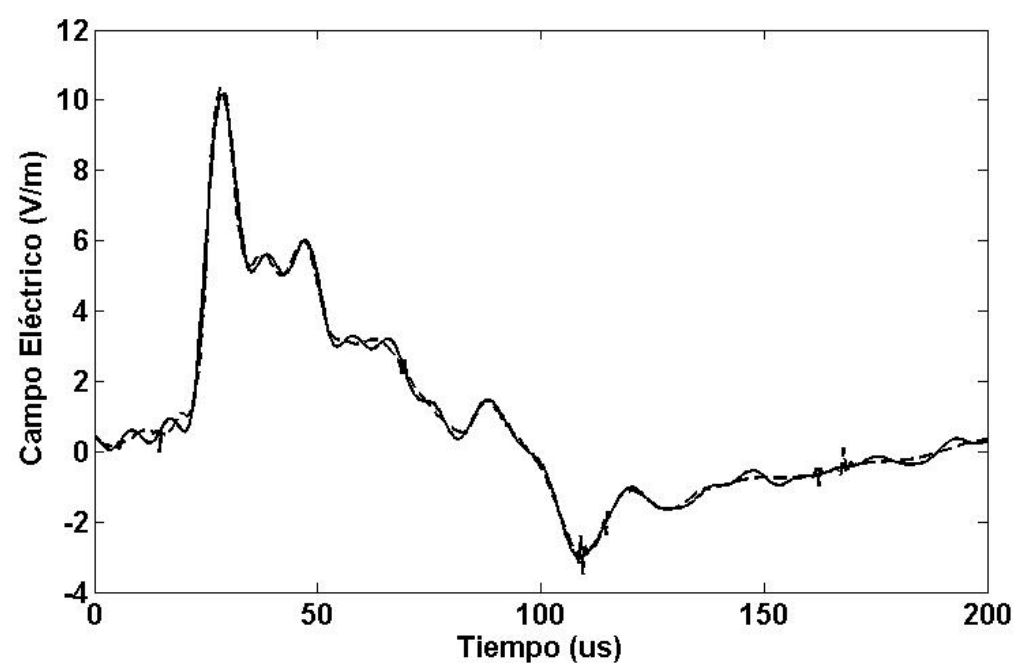

Fig 10: Señal de campo eléctrico (Col2). Línea punteada: filtrada usando transformada wavelet, línea continua: filtrada usando transformada de Fourier 
La Tabla 2 presenta la comparación entre los parámetros de la señal original y las señales filtradas. La diferencia que se observa en el tiempo del frente lento se debe fundamentalmente a que en la señal filtrada usando la transformada de Fourier no es claro el cero de la señal, lo cual lleva a cometer errores en la determinación de dicho parámetro. La principal diferencia se presenta en la estimación del valor máximo de la derivada del campo eléctrico. Esto se debe a que el filtro clásico de Fourier recorta la señal en frecuencias específicas, especialmente altas frecuencias, por lo que además de las señales de ruido es posible que se haya perdido información de la señal de interés en el proceso de filtrado.

Tabla 2: Comparación de los parámetros de la señal de campo eléctrico Col2 filtrada mediante transformada wavelet y transformada de Fourier

\begin{tabular}{|l|c|c|c|c|c|}
\hline Señal Col2 & $\mathrm{F}(\mu \mathrm{s})$ & $\mathrm{R}(\mu \mathrm{s})$ & $\mathrm{CZ}(\mu \mathrm{s})$ & $\operatorname{Emax}(\mathrm{V} / \mathrm{m})$ & $(\partial E / \partial t)_{\max }(\mathrm{V} / \mathrm{m} / \mu \mathrm{s})$ \\
\hline Original & 8.0 & 2.8 & 78.4 & 10.38 & 3.24 \\
\hline Wavelet & 7.6 & 2.9 & 78.4 & 10.14 & 2.45 \\
\hline Fourier & 9.0 & 3.2 & 79.0 & 9.94 & 1,88 \\
\hline
\end{tabular}

Nedjah et al (2010) y Cortés et al (2010) evaluaron el efecto del ruido en las señales de corriente y derivada de corriente y campo eléctrico y derivada de campo eléctrico, respectivamente, encontrando resultados similares en los cuales se observa la alta influencia del ruido en el análisis de las señales de rayo medidas. En los casos que se presentan en este artículo y otros evaluados por los autores se encontró que la técnica de eliminación de ruido basada en la transformada wavelet permite eliminar el ruido de las señales de campo eléctrico de los rayos sin afectar el resto de la señal. A diferencia de los métodos convencionales de filtrado, la técnica aquí empleada no introduce efectos de borde que afecten la respuesta en frecuencia de la señal y por lo tanto su respuesta temporal. Sin embargo, aunque esta técnica tiene importantes beneficios, es necesario compararla con otras técnicas avanzadas. Adicionalmente, se debe garantizar que el procesamiento de las señales se haga sin afectar la información de las mismas para analizar de forma confiable los principales parámetros de las señales de campo eléctrico de los rayos.

En este estudio se pudo observar que el procesamiento de señales empleado simplifica y reduce los posibles errores en el análisis de los parámetros del campo eléctrico del rayo, tal como se presenta en la tabla 1 . Además, en las señales de las figuras $6,7 \mathrm{~b}, 8 \mathrm{~b}$ y $9 \mathrm{~b}$ se evidencia la mejora en la visualización de las características del rayo y en el análisis de los parámetros temporales del campo eléctrico.

\section{CONCLUSIONES}

Para corregir las señales de campo eléctrico es necesario conocer las posibles fuentes de ruido y aplicar procedimientos que lo eliminen sin afectar la señal bajo estudio. Lo anterior se evidenció en este estudio al aplicar la transformada wavelet y encontrar las diferencias entre las señales evaluadas antes y después de su procesamiento, concluyendo que el problema de estimación de parámetros temporales de rayos usando mediciones de campo eléctrico debe seguir siendo estudiado.

Se implementó una metodología para el procesamiento de las señales de campo eléctrico producidas por los rayos, que se fundamenta en la eliminación del ruido presente en dichas señales. Esta metodología facilita la estimación de parámetros temporales fundamentales para el análisis del fenómeno.

El uso de la transformada wavelet es adecuado para el análisis de señales transitorias, como las señales de rayos, ya que realiza un procesamiento en tiempo - frecuencia de manera simultánea, con lo cual se reduce el ruido proveniente de diferentes fuentes sin afectar la señal de interés.

El método empleado para la selección de la wavelet madre se basó en el conocimiento preliminar de la forma de onda del campo eléctrico de los rayos y en la técnica de correlación cruzada. La wavelet madre adecuada para el tratamiento de las señales se seleccionó de un grupo de 24 
wavelet madres tradicionales y se encontró que la que mayor correlación presenta con las señales bajo estudio es la Coiflet4.

A partir de las mediciones de campos eléctricos se extrae información importante para el estudio de protección contra rayos, la evaluación y desarrollo de modelos de la descarga de retorno del rayo y el estudio de la física de la descarga. Sin embargo, puede concluirse que trabajar con señales contaminadas por ruido puede llevar a cometer errores en la evaluación de los parámetros del rayo. Por ejemplo en este artículo se encontraron diferencias entre el $1 \%$ y el 84 $\%$ entre las señales originales y las procesadas, dependiendo del parámetro evaluado.

Aunque por ahora hay pocas aplicaciones relacionadas con la reducción de ruido de las formas de onda del campo eléctrico de los rayos empleando la Transformada Wavelet, este trabajo y otros (Nedjah, 2010) muestran que las posibilidades de esta técnica en la medición y análisis de las señales de los rayos son amplias y aunque en los últimos años se ha avanzado en el área, este es sólo el principio, y aún queda mucho camino por recorrer en este campo.

\section{REFERENCIAS}

Addison, P., The Illustrated Wavelet Transform Handbook: introductory theory and applications in science, engineering, medicine and finance, Institute of Physics Publishing, Taylor \& Francis, USA (2002)

Ahmad, N., M. Fernando, Z. Baharudin, M. Rahman, V. Cooray, Z. Saleh, J. Dwyer, H. Rassoul, The first electric field pulse of cloud and cloud-to-ground lightning discharges, Journal of Atmospheric and Solar-Terrestrial Physics 72 143-150 (2010).

Astafeva, N.M., Wavelet analysis: basic theory and some applications, Physics-Uspekhi, Vol. 39, No 11, 1085-1108 (1996).

Baba, Y., S. Miyazaki y M. Ishii, Reproduction of Lightning Electromagnetic Field Waveforms by Engineering Model of Return Stroke, IEEE Transactions on Electromagnetic Compatibility, 46 (1), 130-133 (2004).

Becerra, M. y V. Cooray, A simplified physical model to determine the lightning upward connecting leader inception, IEEE Transactions on Power Delivery, 21 (2), 897-908 (2006).

Bermudez, J.L., F. Rachidi, W. Janischewskyj, V. Shostak, M. Rubinstein,D. Pavanello, A.M. Hussein, J.S. Chang, M. Paolone, Determination of lightning currents from far electromagnetic fields: Effect of a strike object, Journal of Electrostatics, 65, 289-295 (2007).

Canal, M.R., Comparison of Wavelet and Short Time Fourier Transform Methods in the Analysis of EMG Signals, J Med Syst, 34, 91-94 (2008).

Cooray, V., The Mechanism of the Lightning Flash, In The Lightning Flash, 127-239, IEE Power and Energy Series 34, Londres, Reino Unido (2003).

Cooray, V. y M. Fernando, Lightning Parameters of Engineering Interest, In Lightning Protection, 15-96, IET Power and Energy Series 58, Londres, Reino Unido (2010).

Cortés, C., F. Santamaría, F. Román, F. Rachidi y C. Gomes, Analysis of wavelet based denoising methods applied to measured lightning electric fields, Actas del 33th International Conference on Lightning Protection - ICLP 2010, 1327-1 - 1327-6, Cagliari-Italia, 13 al17 de Septiembre (2010).

Donoho, D. L., De-Noising by Soft-Thresholding, IEEE Transactions on Information Theory, vol. 41, n. 3, 613-627 (1995).

Fernando, M. y V. Cooray, Propagation effect on the electric field time derivatives generated by return strokes in lightning flashes, J. Atmospheric and Solar-Terrestrial Physics, 69 (12), 13881396 (2007). 
Gou, X., M. Chen, Y. Zhang, W. Dong y X. Qie, Wavelet multiresolution based multifractal analysis of electric fields by lightning return strokes, J. Atmospheric Research, 91, 410-415 (2009).

Hussein, A.M., M. Milewski y W. Janischewskyj, Correlating the characteristics of the CN Tower lightning return-stroke current with those of its generated electromagnetic pulse, IEEE Transactions on Electromagnetic Compatibility, 50 (3), 642-650 (2008).

IEC 62305-1, Int. Standard on Protection against lightning - Part 1: General principles, 21-39 (2010).

Islam, M. J. y A. M. Hussein, Frequency domain approach to de-noise the CN Tower lightning derivative signal and its parameters calculations, International Journal of Computer and Electrical Engineering, vol. 1 (3), 328-333 (2009).

Ma, X., C. Zhou, I.J. Kemp, Automated wavelet selection and thresholding for PD detection, IEEE Electrical Insulation Magazine, vol. 18, N. 2, 37-45 (2002).

Mallat, S, A wavelet tour of signal processing. The Sparse Way, Academic Press, USA (2009)

Martínez, A., Z., Pineda y A. Ramos, Ocultamiento del mensaje de señales de voz usando la transformada de ondita Haar discreta, Información Tecnológica, Vol. 21 (3), 135-140 (2010).

Miranda, F.J., Wavelet analysis of lightning return stroke, J. Atmospheric and Solar-Terrestrial Physics, vol.70, 1401-1407 (2008).

Nag, A., B. DeCarlo, V. Rakov, Analysis of microsecond and submicrosecond scale electric field pulses produced by cloud and ground lightning discharges, J. Atmospheric Research, 91, 316-325 (2009).

Nedjah, O., A.M. Hussein, S. Krishnan y R. Sotudeh, Comparative study of adaptive techniques for denoising CN Tower lightning current derivative signals, J. Digital Signal Proc., 20, 607-618 (2010).

Rakov, V.A. y M.A. Uman, Lightning: Physics and Effects, 24-190, Cambridge University Press, Reino Unido (2003).

Ren, H., B. Zhou, V. Rakov, L. Shi, C. Gao, J. Yang, Analysis of lightning-induced voltages on Overhead lines using a 2-d FDTD method And Agrawal coupling model, IEEE Trans. on Electromagnetic Compatibility, vol. 50, no. 3, 651-659 (2008).

Santamaría, F., C. Gomes y F. Roman, Comparison Between the Signatures of Lightning Electric Field Measured in Colombia and that in Sri Lanka, Actas del 28th International Conference on Lightning Protection, 254-256, Kanazawa, Japón, 18 al 22 de Septiembre (2006).

Sharma, S.R., V. Cooray, M. Fernando y F.J. Miranda, Temporal features of different lightning events revealed from wavelet transform, J. Atmospheric and Solar-Terrestrial Physics, 1401-1407, doi:10.1016/j.physletb.2003.10.071 (2010).

Sheshyekani, K., P. Sattari, M. Hazrati, S. Sadeghi y R. Moini, Real-Time Detection of Lightning Electromagnetic Field Data: A Wavelet Approach, Actas de 17th International Zurich Symposium on Electromagnetic Compatibility, Singapur, 27 de Febrero al 3 de Marzo (2006).

Sonnadara, U., V. Cooray y M. Fernando, The lightning radiation field spectra of cloud flashes in the interval $20 \mathrm{kHz}$ to $20 \mathrm{MHz}$, IEEE Trans. on Electromagnetic Compatibility, 48 (1), 234-239 (2006).

Walker, J. S., A Primer on Wavelets and their Scientific Applications, Studies in Advanced Mathematics, Segunda edición, Chapman \& Hall/CRC, USA (2008). 